Volume 1, Number 1, January 2019

\title{
Hypokalemic Periodic Paralysis - A Case Study.
}

\author{
Faisal Ahmed, ${ }^{1}$ Paritosh Kumar Baral, ${ }^{2}$ Sharadindu Shekhar Roy, ${ }^{3}$ Debashish Mohanta ${ }^{4}$
}

\begin{abstract}
Hypokalemic periodic paralysis (HPP) is a rare autosomal dominant channelopathy characterized by muscle weakness or paralysis when there is a fall in potassium levels in the blood. Weakness may be mild and limited to certain muscle groups, or more severe causing full-body paralysis. During an attack, reflexes may be decreased or absent. Attacks may last for a few hours or persist for several days. Recovery is usually sudden when it occurs, due to release of potassium from swollen muscles as they recover. Some patients may develop chronic muscle weakness later in life. Here, we describe a case with history of recurrent muscle weakness who was diagnosed as hypokalemic periodic paralysis.
\end{abstract}

\section{Key Words: Hypokalemic periodic paralysis, Autosomal dominant, Channelopathy}

\section{Introduction}

Periodic paralysis is a group of rare neuromuscular disorders caused by channelopathies. Periodic paralysis is most commonly characterized by hypokalemia due to mutations in sodium or calcium channels. ${ }^{1}$ Hypokalemic periodic paralysis (HPP) is an autosomal dominant channelopathy. In individuals with this mutation, attacks often begin in adolescence and most commonly occur on awakening or after sleep or rest following strenuous exercise high carbohydrate meals, meals with high sodium content, sudden changes in temperature and even excitement. Weakness may be mild and limited to certain muscle groups, or more severe causing full-body paralysis. During an attack reflexes may be decreased or absent. Attacks may last for a few hours or persist for several days. Recovery is usually sudden when it occurs, due to release of potassium from swollen muscles as they recover. Differentiating HPP from secondary hypokalemia is a diagnostic challenge. Some people only develop symptoms of periodic paralysis due to hyperthyroidism. This entity is distinguished with thyroid function tests, and the diagnosis is instead called thyrotoxic periodic paralysis. ${ }^{2}$ Here, we present 1 case with history of recurrent muscle weakness who was diagnosed as hypokalemic periodic paralysis.

\section{Case presentation}

A 50-year-old hypertensive lady with recurrent history of - sudden onset of weakness of both upper and lower limbs. The patient had gone to bed at night with no weakness awoke at late night and was unable to move her upper and lower limbs. She also complaints weakness involved both the proximal and distal muscles. She had no respiratory or swallowing difficulty and was able to move her neck and fascial muscles. She denied any pain or paresthesia. Prior to this episode, the patient had been healthy and denied any recent diarrhea, chest pain, shortness of breath, or weight change. She did not take any medications and denied use of alcohol or drugs or significant changes in diet or activity levels. None of her family members had history of similar type of illness. Physical examination revealed preserved consciousness and orientation with pulse 80 beats/ min, BP- 160/105 $\mathrm{mm}$ of $\mathrm{Hg}$, no jugular venous engorgement, goiter and lymphadenopathy. Cardiovascular system examination revealed no abnormalities and examination of lungs and abdomen were unremarkable. Neurological examination revealed flaccid paralysis of all extremities which involved the proximal and distal muscles with diminished reflexes but all sensory and the cranial nerve functions were intact. Investigations revealed normal hemogram, random blood sugar and creatinine. Serum electrolyte showed potassium level $2.16 \mathrm{mmol} / \mathrm{L}$ with urine spot potassium $10.17 \mathrm{mmol} / \mathrm{L}$. Other serum electrolytes found to be normal. Serum osmolality was $280 \mathrm{mosm} /$ Kg. Electrocardiogram revealed LVH. Ultrasonogram of abdomen shows tiny gall bladder calculi and rest of the findings are normal, CT scan of abdomen and CT scan of brain showed normal findings. After intravenous potassium replacement, the patient's neurologic symptoms resolved completely. Blood pressure was controlled with a combination of Amlodipine and olmesartan. However, the patient has similar episodes of flaccid paralysis accompanying with hypokalemia. Further studies were carried out to determine the cause of recurrent hypokalemia. 24 hours urinary sodium and potassium, serum aldosterone and renin levels were found to be normal. Thyroid stimulating hormone (TSH), free triiodothyronine (FT3) and free thyroxine (FT4) levels were also normal. The patient was diagnosed as hypokalemic periodic paralysis.

\section{Discussion}

\section{Address of correspondence:}

Dr. Faisal Ahmed, Department of Medicine, Z.H Sikder Women's Medical College and Hospital, Dhaka-1209, Bangladesh. Cell: +88 01611 001444. E-mail: faisalahmedjony@gmail.com 
In 1935 the Scottish physician Dr Mary Walker was the first to recognize the association between familial periodic paralysis and hypokalemia ${ }^{3}$. Familial hypokalemic paralysis (FHP) may occur sporadically. Usually, it is a rare autosomal dominant channelopathy in the form of hypokalemic periodic paralysis (HPP). Among the types of periodic paralysis associated with metabolic and electrolyte abnormalities, HPP is the most common with a prevalence of 1 in $100,000^{4}$. HPP is felt to be result of abnormal potassium regulation due to sodium or calcium channel abnormalities._Mutations of the CACNA1S and SCN4A genes have been identified that cause abnormalities in sodium channels resulting in abnormal potassium ion flux ${ }^{5}$. In patients with mutations in CACNA1S or SCN4A, therefore, the channel has a reduced excitability and signals from the central nervous system are unable to depolarize the muscle. As a result, the muscle cannot contract efficiently. The condition is hypokalemic because a low extracellular potassium ion concentration cause the muscle to repolarize to the resting potential more quickly, so even if calcium conductance does occur it cannot be sustained ${ }^{6-8}$. The most striking feature is the sudden onset of weakness ranging in severity from mild, transient weakness to severe disability resulting in life-threatening respiratory failure. As this is primarily a problem with muscle contraction rather than nerve conduction, tendon reflexes may be decreased or absent but sensation is generally intact. Although the serum potassium level is often alarmingly low, other electrolytes are usually normal. Indeed, total body potassium is normal with the change in the serum level reflecting a shift of potassium into cells. Electrocardiographic changes are common, but unlike patients who are truly potassium depleted, the changes do not correlate well with the measured serum level ${ }^{9}$. Differentiating HPP from secondary causes of hypokalemia can present a diagnostic challenge. Hypokalemia most often occurs secondary to dehydration (from chronic diarrhea, chronic laxative abuse, vomiting, or sweating), chronic kidney disease, diabetic ketoacidosis, or renal tubular acidosis ${ }^{10}$. The diagnosis may require an extensive search for the underlying etiology since the treatment varies according to the cause. Thyrotoxic periodic paralysis (TPP) occurs in the setting of hyperthyroidism. The clinical features are similar to those seen with other forms of HPP, but also include the symptoms of thyrotoxicosis. In patients who develop paralysis, however, the symptoms of hyperthyroidism are often quite mild and may be overlooked ${ }^{11}$. Rarely, HPP can result from substantial gastrointestinal or renal potassium losses. In these cases, total body potassium is depleted and requires aggressive replacement. Endocrine abnormalities such as hyperinsulinemia and primary hyperaldosteronism have been associated with $\mathrm{HPP}^{12}$. In our case, all the secondary causes were excluded by both clinically and the necessary investigations. In our case, it was felt in late night at awakening from sleep of which was typical for HPP. The serum potassium level of the was $2.16 \mathrm{mmol} / \mathrm{L}$ and Serum potassium values less than $2.0 \mathrm{meq} / \mathrm{L}$ often suggesting secondary causes of hypokalemia ${ }^{13}$. The initial presentation of our cases with absent signs of secondary causes of hypokalemia, as well as the accompanying symptoms of this patient suggest HPP to be the most likely diagnosis. The recurrence of paralytic attack is also suggestive of HPP which were present in our cases. Autosomal dominant pattern of family history of our second case guided us positively to make this type of diagnosis in that patient also. In an acute paralytic attack, intravenous and oral potassium with 24-hours cardiac monitoring for rebound hyperkalemia are recommended for the management and were performed in our two patients. When a patient has persistent paralytic attacks, preventive lifestyle modifications have been suggested including avoidance of vigorous exercise and reduction in dietary carbohydrates. If lifestyle modifications are not effective, the clinician should consider adding medications. Oral potassium supplementation, acetazolamide and spironolactone have been shown to reduce frequency of attacks ${ }^{14}$. Our patients were described these things properly and were of our regular follow-up for further needful actions as recommendation.

\section{Conclusion}

In a patient with sudden onset of paralysis, especially those with no history or evidence of other diseases and no significant risk factors for stroke and there is recurrence of such reversible phenomenon, HPP is important to consider in initial work-up. So, high index of suspicion is necessary for making a diagnosis of HPP in an acute attack of paralysis with low serum potassium having no secondary causes of hypokalemia. In this case study, the patient presented with sudden onset of flaccid paralysis involving both sides of the body and markedly low serum potassium with normal TSH, T3 and T4 levels. The presentation was typical of HPP. The paralysis resolved completely following potassium replacement. Without proper diagnosis and treatment HPP can be fatal. Correction of potassium abnormalities can resolve the symptoms quickly and completely. Physicians should have appropriate attention to diagnose timely and manage such cases.

\section{Reference}

1. Matthews E, Labrum R, Sweeney MG, et al. Voltage sensor charge loss accounts for most cases of hypokalemic periodic 


\section{Volume 1, Number 1, January 2019}

paralysis. Neurology 2009; 72(18): 1544-1547.

2. Okinaka S, Shizume K, Iino S, et al. The association of periodic paralysis and hyperthyroidism in Japan. J Clin Endocrinol Metab 1957; 17(12):1454-1459.

3. Aitken RS, Allot EN, Gastelden LI, Walker MB. Observations on a case of familial periodic paralysis. Clin Sci 1937; 3: 47-57.

4. Fontaine B, Vale-Santos J, Jurkat-Rott K, et al. Mapping of the hypokalaemic periodic paralysis (HypoPP) locus to chromosome 1q31-32 in three European families. Nat Genet 1994; 6(3):267272.

5. Wang W, Jiang L, Ye L, et al. Mutation screening in Chinese hypokalemic periodic paralysis patients. Mol Genet Metab 2006; 87(4):359-363.

6. Rüdel R, Lehmann-Horn F, Ricker K, Küther G. Hypokalemic periodic paralysis: in vitro investigation of muscle fiber membrane parameters. Muscle Nerve 1984; 7 (2): 110-120.

7. Jurkat-Rott K, Lehmann-Horn F. Muscle channelopathies and critical points in functional and genetic studies. J. Clin. Invest 2005; 115 (8): 2000-2009.

8. Kim SJ, Lee YJ, Kim JB. Reduced expression and abnormal localization of the KATP channel subunit SUR2A in patients with familial hypokalemic periodic paralysis. Biochemical and Biophysical Research Communications 2010; 391: 974-978.

9. Soule BR, Simone NL._Hypokalemic Periodic Paralysis: a case report and review of the literature. Cases Journal 2008; 1: 256.

10. Kumar KK, Kadhim Z, Mohan JK. Acquired non-thyrotoxic hypokalemic periodic paralysis: A case report. Edorium J Neurol 2016; 3:1-3.

11. Kung AW. Clinical review: Thyrotoxic periodic paralysis: a diagnostic challenge. J Clin Endocrinol Metab 2006; 91(7):24902495.

12. Ogawa T, Kamikubo K. Hypokalemic periodic paralysis associated with hypophosphatemia in a patient with hyperinsulinemia. Am J Med Sci 1999; 318(1):69-72.

13. Miller TM, Dias da Silva MR, Miller HA. Correlating phenotype and genotype in the periodic paralyses. Neurology 2004; 63(9):1647-1655.

14. Fiore DM, Strober JB. Treatment of hypokalemic periodic paralysis with topiramate. Muscle Nerve. 2011; 43(1): 127-129. 